\title{
THE COMPOSITION OF OPERATOR-VALUED MEASURABLE FUNCTIONS IS MEASURABLE
}

\author{
A. BADRIKIAN, G. W. JOHNSON, AND IL YOO
}

(Communicated by Palle E. T. Jorgensen)

\begin{abstract}
Given separable Frechet spaces, $E, F$, and $G$, let $\mathscr{L}(E, F)$, $\mathscr{L}(F, G)$, and $\mathscr{L}(E, G)$ denote the space of continuous linear operators from $E$ to $F, F$ to $G$, and $E$ to $G$, respectively. We topologize these spaces of operators by any one of a family of topologies including the topology of pointwise convergence and the topology of compact convergence. We will show that if $(X, \mathscr{F})$ is any measurable space and both $A: X \rightarrow \mathscr{L}(E, F)$ and $B: X \rightarrow$ $\mathscr{L}(F, G)$ are Borelian, then the operator composition $B A: X \rightarrow \mathscr{L}(E, G)$ is also Borelian. Further, we will give several consequences of this result.
\end{abstract}

\section{INTRODUCTION}

A special case of the result discussed in the abstract was proved in [10]. The present setting is far more general. The motivation for [10] came from work on [3] and on a related longer paper [4] which is still in preparation. Briefly, these papers use the heuristic ideas of Feynman's operational calculus for noncommuting operators to develop perturbation series which may be applicable in a variety of settings. Products of strong operator measurable functions appear repeatedly in [3, 4], and Theorem 5 of [10] (the main result) guarantees that these products are themselves strong operator measurable so that the power of the Bochner integral can be employed.

Similar questions involving perturbation series arose in earlier work on the Feynman integral, but there the strong operator measurability of the products could be argued without establishing a general theorem. One consequence, however, of [10] is that substantially shorter proofs of these theorems on the Feynman integral can now be given. References to the relevant earlier papers are provided in [10] and will not be repeated here.

The keys to [10] are

(i) the concept of a Lusin $\mu$-measurable function on $X$, where $X$ is a Hausdorff topological space and $\mu$ is a finite Radon measure on $X$,

Received by the editors March 8, 1993 and, in revised form, October 4, 1993.

1991 Mathematics Subject Classification. Primary 46G10, 28B05; Secondary 47B10, 46A04.

Key words and phrases. Lusin space, Souslin space, operator-valued measurable function, measurability of operator composition, strong operator measurability, Frechet space, Banach space, topology of simple convergence, topology of compact convergence.

Albert Badrikian died in the summer of 1994. His coauthors dedicate their work on this subject to his memory. 
and

(ii) the fact that $\mathscr{L}(H)$, under the strong operator topology, is a "Lusin space" [14, Theorem 7, pp. 112-114].

In the present paper, (i) plays no role; $(X, \mathscr{F})$ is an abstract measurable space. On the other hand, the more general results of this paper do depend in an essential way on an even fuller exploitation (using various results from [1]) of the fact that the spaces of operators involved are Lusin spaces in any of the topologies under consideration. The books $[5,6,15]$ are additional good references on these and related measure-theoretic matters.

The increased generality of our present paper permits further applications. We first mention two extensions of the earlier applications. (These will not be repeated in the main body of this paper.)

The presence of a separable Hilbert space $H$ in [10] was motivated by [3, 4] which are directed primarily toward quantum models. Other physical problems, reaction-diffusion phenomena, for example, suggest the use of a variety of separable Banach spaces other than $H$. Although work on such extensions is just beginning, it already follows from the results of this paper that the products of operators which form the integrands of the perturbation series involved are measurable. Thus, one can at least get started.

The earlier work on the Feynman integral which involved perturbation series and which required proofs of strong operator measurability was primarily in the setting of the Hilbert space $L^{2}\left(\mathbb{R}^{v}\right)$. However, [11] involved $L^{p}\left(\mathbb{R}^{v}\right)$ and $L^{p^{\prime}}\left(\mathbb{R}^{v}\right)$, where $1<p \leq 2$ and $1 / p+1 / p^{\prime}=1$. The results of this paper yield the necessary measurability immediately and so shorten the proof of $[11$, Theorem 2.1, especially pp. 107-108].

We close this introduction by clarifying terminology which we sometimes use. Let $(X, \mathscr{F})$ be a measurable space, and let $Y$ be a topological space. We say that $f: X \rightarrow Y$ is Borelian provided that the inverse image of every Borel set in $Y$ is in $\mathscr{F}$. We reserve the term Borel measurable for the case where $X$ is also a topological space, $\mathscr{F}$ is the Borel class of $X$, and $f$ is Borelian.

\section{THE MAIN RESULT AND SOME COROLLARIES}

The scalar field for all the vector spaces that we will consider can be either $\mathbb{R}$ or $\mathbb{C}$, the real or complex numbers, respectively. Our primary concern at first will be with the topology of pointwise or simple convergence on the spaces of operators. The space $\mathscr{L}(E, F)$, equipped with the topology of simple convergence, will be denoted $\mathscr{L}_{S}(E, F)$.

The proof of our main result depends on some facts about Lusin spaces. First, we review the following definitions.

Definition 1. Let $Z$ be a Hausdorff topological space.

(i) $Z$ is a Polish space if and only if it is separable and can be metrized by means of a complete metric.

(ii) $Z$ is Lusin if and only if it is the image of a Polish space under a continuous bijection.

(iii) $Z$ is Souslin if and only if it is the image of a Polish space under a continuous surjection. 
A simple fact which will be useful to us is that every Hausdorff topology which is weaker than a Lusin topology is also a Lusin topology. It is clear that every Polish space is Lusin, and every Lusin space is Souslin.

Theorem 1. (A) [1, Corollary 2, p. 121] If $\tau_{1}$ and $\tau_{2}$ are two comparable Lusin topologies on $Z$, then the corresponding Borel $\sigma$-fields coincide.

(B) [1, Proposition 9, p. 124] Let $Z$ be a Lusin space, and suppose that $\left\{f_{n}\right\}$ is a sequence of Borel measurable scalar-valued functions on $Z$ which separates the points of $Z$. Then the $\sigma$-field generated by the functions $\left\{f_{n}\right\}$ is identical with the Borel $\sigma$-field of $Z$.

(C) [1, Proposition 15, p. 138] Let $Z$ and $Y$ be a Lusin space and Hausdorff topological space, respectively, and suppose that $\left\{f_{i}\right\}_{i \in I}$ is a family of continuous mappings from $Z$ into $Y$ which separates the points of $Z$. Then there exists a countable subset $D$ of $I$ such that $\left\{f_{i}\right\}_{i \in I}$ separates the points of $Z$.

Corollary 1. Let the hypotheses of Theorem 1(C) hold but with $Y$ equal to the scalar field. Then the $\sigma$-field generated by the family of functions $\left\{f_{i}\right\}_{i \in I}$ is identical to the Borel $\sigma$-field of $Z$.

Proof. Applying (C) and (B) of Theorem 1 we have

$$
\mathscr{B}(Z)=\sigma\left\{f_{i}: i \in D\right\} \subset \sigma\left\{f_{i}: i \in I\right\} \subset \mathscr{B}(Z) \text {. }
$$

Theorem 1 remains true if "Lusin" is replaced by "Souslin" throughout; in fact, these results are given in [1] for this larger class of spaces. Corollary 1 then holds as well for $Z$ a Souslin space.

The assumption of continuity is essential in Theorem $1(C)$. The characteristics functions $\chi_{\{z\}}, z \in Z$, are Borel measurable, and the family $\left\{\chi_{\{z\}}: z \in Z\right\}$ is separating. However, if $Z$ is not countable, we cannot extract a countable subfamily that is separating.

Given locally convex spaces $E$ and $F$, we let $\mathscr{L}_{c}(E, F)$ denote the space $\mathscr{L}(E, F)$ equipped with the topology of uniform convergence on compact subsets of $E$.

Theorem 2. Let $E, F$, and $G$ be three separable Frechet spaces, and let $(X, \mathscr{F})$ be a measurable space. Further, let $A: X \rightarrow \mathscr{L}(E, F)$ and $B: X \rightarrow \mathscr{L}(F, G)$ be two measurable mappings with respect to $\mathscr{F}$ and the Borel $\sigma$-fields of $\mathscr{L}_{s}(E, F)$ and $\mathscr{L}_{s}(F, G)$, respectively.

Then the map $x \mapsto B(x) A(x)$ is also measurable from $X$ into $\mathscr{L}(E, G)$ with respect to $\mathscr{F}$ and the Borel $\sigma$-field of $\mathscr{L}_{s}(E, G)$.

Proof. Given $\left(e, g^{\prime}\right) \in E \times G^{\prime}$, we denote by $\ell_{e}, g^{\prime}$ the linear form on $\mathscr{L}(E, G)$ defined by

$$
\ell_{e, g^{\prime}}(u):=\left\langle u(e), g^{\prime}\right\rangle_{G, G^{\prime}} .
$$

It is easy to check that $\ell_{e}, g^{\prime}$ is continuous on $\mathscr{L}_{s}(E, G)$. Also, it follows from the Hahn-Banach Theorem that the family $\left\{\ell_{e}, g^{\prime}: e \in E, g^{\prime} \in G^{\prime}\right\}$ separates the points of $\mathscr{L}_{s}(E, G)$.

A key fact for us [14, Theorem 7, p. 112] is that the space $\mathscr{L}_{c}(E, F)$ is a Lusin space. Since the topology of simple convergence is Hausdorff and is a weaker topology than the Lusin topology of $\mathscr{L}_{c}(E, F)$, it follows that $\mathscr{L}_{s}(E, F)$ is also a Lusin space. The same is true, of course, for $\mathscr{L}_{s}(F, G)$ and $\mathscr{L}_{s}(E, G)$.

Now applying Corollary 1 , we see that $\sigma\left\{\ell_{e}, g^{\prime}: e \in E, g^{\prime} \in G^{\prime}\right\}$ coincides with the Borel $\sigma$-field of $\mathscr{L}_{s}(E, G)$. Hence, to finish our proof, it suffices 
to show that $\left\langle B(\cdot) A(\cdot)(e), g^{\prime}\right\rangle_{G, G^{\prime}}$ is measurable for every $e \in E$ and $g^{\prime} \in$ $G^{\prime}$. But $\left\langle B(\cdot) A(\cdot) e, g^{\prime}\right\rangle_{G, G^{\prime}}=\left\langle A(\cdot) e, B^{*}(\cdot) g^{\prime}\right\rangle_{F, F^{\prime}}$, where $B^{*}(x)$ denotes the transpose of the operator $B(x)$. Hence, it suffices to show that

$$
\left\langle A(\cdot) e, B^{*}(\cdot) g^{\prime}\right\rangle_{F, F^{\prime}} \quad \text { is measurable for every } e \in E \text { and } g^{\prime} \in G^{\prime} .
$$

But $\left\langle f, B^{*}(\cdot) g^{\prime}\right\rangle_{F, F^{\prime}}=\left\langle B(\cdot) f, g^{\prime}\right\rangle_{G, G^{\prime}}$ is measurable for every $f \in F$. Further, for every $e \in E$, the map $A(\cdot) e$ is scalarly measurable; that is, $\left\langle A(\cdot) e, f^{\prime}\right\rangle_{F, F^{\prime}}$ is measurable for every $f^{\prime} \in F^{\prime}$. Since $F$ is metrizable and separable, it follows that $A(\cdot) e$ is Bochner measurable; that is, $A(\cdot) e$ is the limit of a sequence of elementary measurable functions $\left\{\psi_{k}\right\}$, where each $\psi_{k}$ has the form

$$
\psi_{k}(\cdot)=\sum_{j=1}^{n_{k}} \chi_{A_{j}^{k}}(\cdot) a_{j}^{k}, \quad A_{j}^{k} \in \mathscr{F}, a_{j}^{k} \in F .
$$

From this, we see that

$$
\left\langle A(\cdot) e, B^{*}(\cdot) g^{\prime}\right\rangle_{F, F^{\prime}}=\lim _{k \rightarrow \infty} \sum_{j=1}^{n_{k}} \chi_{A_{j}^{k}}(\cdot)\left\langle a_{j}^{k}, B^{*}(\cdot) g^{\prime}\right\rangle_{F, F^{\prime}}
$$

is measurable. Thus (1) is verified and the proof is complete.

The following corollary comes immediately from Theorems $1(\mathrm{~A})$ and 2.

Corollary 2. Let $E, F$, and $G$ be three separable Frechet spaces, and let $(X, \mathscr{F})$ be a measurable space. Topologize each of $\mathscr{L}(E, F), \mathscr{L}(F, G)$, and $\mathscr{L}(E, G)$ with any Lusin topology which is comparable with the topology of simple convergence. (We need not make the same choice for all three spaces of operators.) Further, let $A: X \rightarrow \mathscr{L}(E, F)$ and $B: X \rightarrow \mathscr{L}(F, G)$ be two measurable mappings with respect to $\mathscr{F}$ and the Borel $\sigma$-fields of $\mathscr{L}(E, F)$ and $\mathscr{L}(F, G)$, respectively.

Then the map $x \mapsto B(x) A(x)$ is also measurable from $X$ into $\mathscr{L}(E, G)$ with respect to $\mathscr{F}$ and the Borel $\sigma$-field of $\mathscr{L}(E, G)$.

The topologies of (i) simple convergence, (ii) uniform convergence on compact sets, and (iii) uniform convergence on compact, convex, circled sets are all of importance in the study of operators on locally convex spaces [13] and are all allowable choices in Corollary 2. The topology of (iv) uniform convergence on compact sets having a countable cardinal number is another permissible choice.

It may not be immediately transparent that Theorem 2 includes Theorem 5 from [10]. We give a further corollary to clarify this point.

Corollary 3. Let $(X, \mathscr{L}, \mu)$ be a finite, complete measure space, and let $E$ be a separable Banach space. Finally, suppose that $A, B: X \rightarrow \mathscr{L}(E)$ are both strong operator measurable in the sense of Hille and Phillips (see [9, p. 72] or [10]).

Then $B A: X \rightarrow \mathscr{L}(E)$ is also strong operator measurable in the same sense. Proof. This result follows from Theorem 2 and the fact that if $(X, \mathscr{L}, \mu)$ is any finite, complete measure space and $E$ is a separable Banach space, then strong operator measurability in the sense of Hille and Phillips is equivalent to $\mathscr{L}-\mathscr{B}\left(\mathscr{L}_{s}(E)\right)$ measurability. The Hilbert space case of the assertion just made is proved in Theorem 2 of [10]. The extension to a separable Banach 
space is rather straightforward and will be omitted. (For the reader who wishes to check the details, we remark that

(i) part (a) of [12, Proposition p. 116] is done for a separable Banach space, and

(ii) Theorem IV.22 [12, p. 116] is valid in that setting as noted on p. 119 of [12].

Finally,

(iii) the Hahn-Banach Theorem yields an appropriate replacement for the rank-one operator used in the proof of [10, Proposition 1]).

Corollary 3 clearly contains Theorem 5 from [10] as a very special case.

Theorem 2 fills a gap in the literature, but its proof is not especially difficult when approached from the right direction. We have already found this result and its corollaries useful, and we suspect others will also. In further applications, it may sometimes be helpful to know additional properties of Lusin and Souslin spaces. We illustrate this by stating a final corollary which is easily proved using the following facts:

(A) [1, Corollary 1, p. 122] A subspace $Y$ of a Lusin topological space $Z$ is itself Lusin if and only if it belongs to the Borel $\sigma$-field of $Z$.

(B) [1, Corollary 3, p. 122-123] Let $f$ be a continuous injection of a Lusin space $Z$ into a Hausdorff space $W$. Then the image $f(B)$ of every Borel subset of $Z$ is a Borel subset of $W$.

Now let $H_{1}, H_{2}$, and $H_{3}$ be separable Hilbert spaces, and let $(X, \mathscr{F})$ be a measurable space. Also let $\left(\mathscr{L}^{1}\left(H_{2}, H_{3}\right),\|\cdot\|_{1}\right)$ and $\left(\mathscr{L}^{2}\left(H_{2}, H_{3}\right),\|\cdot\|_{2}\right)$ denote the trace class and Hilbert-Schmidt operators, respectively, from $\mathrm{H}_{2}$ to $H_{3}$ with their appropriate norms. (See $[7,8,12]$ for material on these spaces.)

Let $i \in\{1,2\}$. If $A: X \rightarrow \mathscr{L}_{s}\left(H_{1}, H_{2}\right)$ and $B: X \rightarrow \mathscr{L}^{i}\left(H_{2}, H_{3}\right)$ are two measurable mappings with respect to $\mathscr{F}$ and the Borel $\sigma$-fields of $\mathscr{L}_{s}\left(H_{1}, H_{2}\right)$ and $\left(\mathscr{L}^{i}\left(H_{2}, H_{3}\right),\|\cdot\|_{i}\right)$, respectively, then the map $B \circ A: X \rightarrow \mathscr{L}^{i}\left(H_{1}, H_{3}\right)$ is measurable with respect to $\mathscr{F}$ and the Borel $\sigma$-field on $\left(\mathscr{L}^{i}\left(H_{1}, H_{3}\right),\|\cdot\|_{i}\right)$.

\section{REFERENCES}

1. A. Badrikian, Séminaire sur les fonctions aléatoires et les measures cylindriques, Lecture Notes in Math., vol. 139, Springer, Berlin, 1970.

2. D. L. Cohn, Measure theory, Birkhäuser, Boston, 1980.

3. B. DeFacio, G. W. Johnson, and M. L. Lapidus, Feynman's operational calculus as a generalized path integral, Stochastic Processes: A Festschrift in Honour of Gopinath Kallianpur, Springer-Verlag, New York, 1993, pp. 51-60.

4. __ Feynman's operational calculus and evolution equations (in preparation).

5. N. Dinculeanu, Vector measures, Pergamon Press, London and New York, 1967.

6. Integration on locally compact spaces, Noordhoff, Leyden, the Netherlands, 1974.

7. N. Dunford and J. T. Schwartz, Linear operators, Part II, Wiley Interscience, New York, 1963.

8. I. M. Gelfand and N. Ya. Vilenkin, Generalized functions, Vol. 4, Applications of Harmonic Analysis, Academic Press, New York, 1964.

9. E. Hille and R. S. Philips, Functional analysis and semi-groups, rev. ed., Amer. Math. Soc. Colloq. Publ., vol. XXXI, Amer. Math. Soc., Providence, RI, 1957.

10. G. W. Johnson, The product of strong operator measurable functions is strong operator measurable, Proc. Amer. Math. Soc. 117 (1993), 1097-1104. 
11. G.W. Johnson and D. L. Skoug, The Cameron-Storvick function space integral: an $\mathscr{L}\left(L_{p}, L_{p^{\prime}}\right)$ theory, Nagoya Math. J. 60 (1976), 93-137.

12. M. Reed and B. Simon, Methods of modern mathematical physics, Vol. I, rev. and enl. ed., Functional Analysis, Academic Press, New York, 1980.

13. H. H. Schaefer, Topological vector spaces, Macmillan, New York, 1966.

14. L. Schwartz, Radon measures on arbitrary topological spaces and cylindrical measures, Oxford Univ. Press, London, 1973.

15. M. Sion, A theory of semigroup valued measures, Lecture Notes in Math., vol. 355, SpringerVerlag, New York, 1973.

Mathematiques Appliquees, Universite de Clermont, Complexe Scientifique des Cézeaux, 63177 Aubiére, Cédex, France

Department of Mathematics and Statistics, University of Nebraska-Lincoln, LinCOLN, NEBRASKa 68588-0323

Department of Mathematics, Yonsei University, KANGWONDo 222-701, Korea 